\title{
The Absolute Frame of Reference
}

\author{
Ninh Khac Son
}

Date Performed: 05 March 2020

\begin{abstract} calculation as follows:

$$
\begin{aligned}
& v_{\text {Earth }}=1.852819296 * 10^{8} \mathrm{~m} / \mathrm{s} \\
& C_{\max }=4.8507438399 * 10^{8} \mathrm{~m} / \mathrm{s}
\end{aligned}
$$
\end{abstract}

The manuscript found the formula to calculate the real velocity of the earth and the maximum velocity in the universe, the values of the quantities after

In order to calculate the above results, the manuscript has built a reference frame transformation suitable for all types of motion(suitable for both linear motion and chaotic motion of the reference frame). This means that we will calculate the velocity of an object without using the distance $\mathrm{S}$ quantity of the object.

Key words: Frame of reference; The speed of the Earth; Maximum speed.

\section{Methodology}

\subsection{The law of conservation of time}

The law of conservation of time mentioned in the immobility theory, it describes the total time $\mathrm{T}$ of a moving object in space in the form:

$$
t^{\prime}+t=T
$$

Where:

- $\mathrm{T}$ is the intermittent motion time of the object (total time), the value of $\mathrm{T}$ is preserved.

- t' is the motion time with the speed $C_{\max }$ of the object.

- $\mathrm{t}$ is the absolute immobility time of the object.

Reference source: Section 1 of the Immobility theory. 


\subsection{The methods of calculating the average velocity}

We usually calculate the velocity of a moving object in nature by the formula:

$$
v=\frac{S}{t}=\frac{\text { value_of_distance }}{\text { immobility_time }}
$$

But in immobility theory, the velocity of an object will be calculated by the formula:

$$
v=C_{\max } * \frac{t^{\prime}}{T}
$$

Therefore, the value of distance $\mathrm{S}$ in the immobility theory does not make much sense, it is only a resultant value, not a causal value. This is very important for us to build a uniform frame of reference for physical equations without classifying the frame of reference (inertia, non-inertia, quantum reference system, ...).

Note:

- If we consider the earth to be a stand still and we compare the velocities of two objects then the calculated velocity value is only a difference of the velocity of the two objects (not the real velocity of the objects). Therefore, the formula for calculating the correct velocity must always be compared with an absolute frame of reference, so equation (1) must be $v=\frac{S}{T}=\frac{\text { value_of_distance }}{\text { total_time }}$.

- But because we have not determined the absolute frame of reference, so we have equated the total time $T$ of the earth with the immobility time $t$ of the earth $T=t$ to build a relative reference frame, this leads to equation (1) as mentioned.

- The contents of the manuscript will build an absolute frame of reference, which will then determine motion time $t$ ' of the earth in a unit of time $T$ given for survey.

\subsection{Two basic math problems}

When comparing the method of calculating the velocity of an object according to equations (1) and (2), we see a little difference as follows:

- For the method of calculating the velocity according to classical physics (Eq:1), we need to know the value of distance $\mathrm{S}$ of the object, then calculate the velocity value. If the object moves without determining the distance 
value $\mathrm{S}$ (chaotic motion) then it is very difficult for us to calculate the average velocity while the object has not finished its motion journey. Therefore, it is imperative that we build and classify different reference frames to suit each category of motion(inertial reference frame, non-inertial reference frame, quantum reference frame,...).

- But the method of calculating the velocity according to the law of conservation of time $(\mathrm{Eq}: 22$ will solve these disadvantages of classical physics.

More specifically, we will solve the number 1 problem as follows:

Topic of problem 1:

You stand still to calculate my velocity, while I move from position A to B on a chaotic graph(motion orbit is unknown). The time displayed on your watch and me(two clocks) start working from the moment I move and stop counting the time when I get to position B.(Figure 1) 


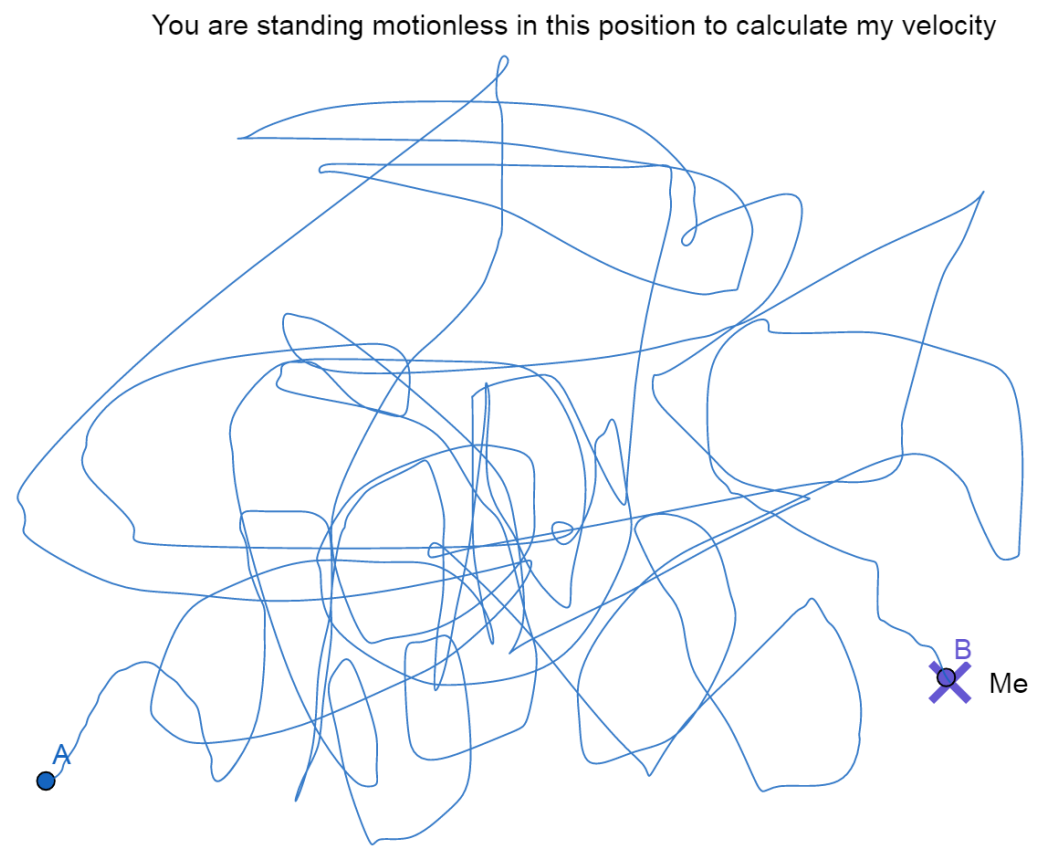

I move from $A$ to $B$ according to the graph with the blue border

(Figure 1: Calculate the velocity of an object with an unknown motion orbit)

Question:

How would you calculate my velocity in this case when my distance value is unknown?

\section{Answer:}

We will not be able to solve this problem using the method of classical physics, because $\mathrm{S}$ is indeterminate. But if we use the method of calculating velocity according to the immobility theory then the problem will become simple as follows:

You take the time value displayed on your watch and then subtract the time value displayed on my watch, this value is my motion time $t_{m e}^{\prime}$.

$$
t_{\text {you }}-t_{m e}=\Delta t=t_{m e}^{\prime}
$$


Therefore, my velocity is determined by the expression:

$$
\begin{gathered}
v_{m e}=C_{\max } * \frac{t_{m e}^{\prime}}{T} \approx C_{\max } * \frac{t_{m e}^{\prime}}{t_{\text {you }}} \\
\Rightarrow S_{m e}=v_{m e} * T \approx v_{m e} * t_{\text {you }}
\end{gathered}
$$

Note:

- The approximation sign $(\approx)$ indicates that we are using a relative frame of reference (the earth) that is not an absolute frame of reference.

- The number 1 problem is considered the basis for us to build the method of calculating the velocity without necessarily having the value of the distance $S$ of the object. Specifically in this manuscript, we will calculate the actual velocity of the earth without knowing the orbit or value $S$ of the earth.

Continuing to develop from problem 1, we build problem 2 to contact a chaotic frame of reference.

Topic of problem 2:

You and I both calculate the velocity of a moving M object, you stand still and I move in an indefinite graph as shown in Figure 2. Your watch and my watch start when the $\mathrm{M}$ object moving from position $\mathrm{A}$, and they stop working when object $\mathrm{M}$ reaches position $\mathrm{B}$. 


\section{$X$ You}

You stand still to calculate the velocity of the object M

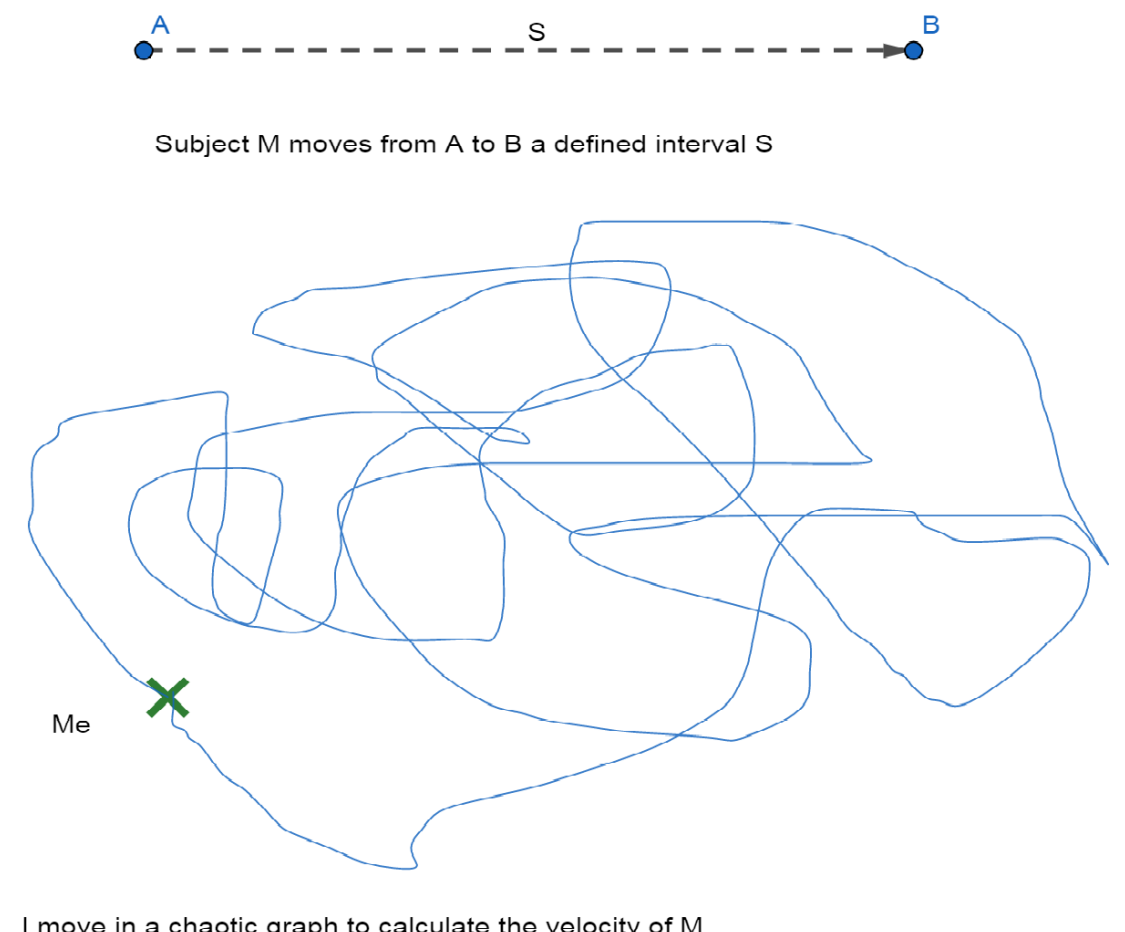

(Figure 2: Which frame of reference transformation is used to synchronize time with a chaotic frame of reference?)

Question:

Assuming the distance $\mathrm{S}$ of the object $\mathrm{M}$ is deterministic, then both you and I can calculate the velocity of the object M. But my calculation results are different from your results, and we will use the Which method of transforming the frame of reference to match these values?

Answer: 
There is no answer in classical physics, because both frame of reference transformations (Galilean transformation and Lorentz transformation) are inertial transformations.

Therefore, the manuscript will construct a frame of reference transformation regardless of the motion orbits of the frame of reference, which means the manuscript will build a proper generalized frame of reference transformation with every kind of movement of the frame of references.

And the answer to problem 2 will be solved simply after we build the frame of reference transformation.

\section{LCT-transformation}

This transformation is based on the Law of Conservation of Time, so we name this transformation method LCT-transformation.

2.1. Describe the frame of reference based on the law of conservation of time Each moving object in space can be understood that it always has a frame of reference that corresponds to it. Based on the law of conservation of time to investigate the movement of these frames of reference in space, we have:

For any frame of reference $\mathbf{i}$ (object i), it has three types of time:

- $T_{i}$ is the total time of the frame of reference i, $T_{i}$ is preserved by the formula: $T_{i}=t_{i}^{\prime}+t_{i}$

- $t_{i}^{\prime}$ is the motion time of the frame of reference $\mathrm{i}$ in $T_{i}$

- $t_{i}$ is the immobility time of the frame of reference i in $T_{i}, t_{i}$ is the only type of time displayed on the physical clock $\left(T_{i}\right.$ and $t_{i}^{\prime}$ are not displayed on the clock).

When surveying multiple frames of reference at the same time $1,2, . ., \mathrm{i}, . ., \mathrm{n}$, the total time of these frames of reference is equal: 


$$
T_{1}=T_{2}=T_{3}=\ldots=T_{i}=\ldots=T_{n}=T
$$

\subsection{Methods of constructing LCT-transformation}

Investigating the motion of two objects A and B in space, two objects A and $\mathrm{B}$ are considered as the two reference frames $\mathrm{A}$ and $\mathrm{B}$.

Consider case 1: $\mathrm{A}$ is absolute immobility and $\mathrm{B}$ moves.

In this case, we have the time variables corresponding to A and B as follows:

$$
\left\{\begin{array}{l}
t_{A}=T_{A}=T \\
t_{B}=T_{B}-t_{B}^{\prime}=T-t_{B}^{\prime}
\end{array}\right.
$$

Therefore:

$$
\frac{t_{B}}{t_{A}}=\frac{T-t_{B}^{\prime}}{T}=1-\frac{t_{B}^{\prime}}{T}=1-\frac{v_{B}}{C_{\max }}=\beta_{1}
$$

Conclusion: In case 1, the LCT-transformation has the form:

$$
\left\{\begin{array}{l}
t_{B}=\beta_{1} * t_{A} \\
\beta_{1}=1-\frac{v_{B}}{C_{\max }}
\end{array}\right.
$$

Consider case 2: A stands still on the surface of the earth, B moves in a chaotic motion on the surface of the earth.

Therefore:

$$
\left\{\begin{array}{l}
t_{A}=T-t_{A}^{\prime}=T-t_{0}^{\prime} \\
t_{B}=T-t_{B}^{\prime}=T-t_{0}^{\prime}-\Delta t^{\prime} \\
t_{0}^{\prime}=t_{\text {Earth }}^{\prime} \\
\Delta t^{\prime}=t_{B}^{\prime}-t_{A}^{\prime}=t_{A}-t_{B}
\end{array}\right.
$$




$$
\Rightarrow \frac{t_{B}}{t_{A}}=\frac{T-t_{0}^{\prime}-\Delta t^{\prime}}{T-t_{0}^{\prime}}=1-\frac{\Delta t^{\prime}}{T-t_{0}^{\prime}}=1-\frac{v_{B}-v_{0}}{C_{\max }-v_{0}}=\beta_{2}
$$

Conclusion: In case 2, the LCT-transformation has the form:

$$
\left\{\begin{array}{l}
t_{B}=\beta_{2} * t_{A} \\
\beta_{2}=1-\frac{v_{B}-v_{0}}{C_{\max }-v_{0}}
\end{array}\right.
$$

\section{The comparison in two cases:}

The transformation of the frame of reference in two cases has the same structure, but the values $\beta_{1}$ and $\beta_{2}$ are different. $\beta_{1}$ and $\beta_{2}$ are equal if and only if the velocity $v_{B}$ of $\mathrm{B}$ is equal to the maximum velocity $C_{\max }$

$$
\beta_{1}=\beta_{2} \Leftrightarrow \frac{v_{B}}{C_{\max }}=\frac{v_{B}-v_{0}}{C_{\max }-v_{0}} \Leftrightarrow v_{B}=C_{\max }
$$

Therefore, if we use the transformation in case 2 then the result will have errors, which means that the transformations in both cases are theoretically correct but the transformation in the case 2 is only applied when the velocity $v_{B}$ of reference frame $\mathrm{B}$ reaches a maximum: $v_{B}=C_{\max }$.

In other words: We need to find the initial velocity $v_{0}$ (the real velocity of the earth, the real velocity of you and mine, ...) to bring the problems to case 1. And if we can determine $v_{0}$ then we also should not use LCT-transformation in case 2 to calculate in quantum mechanics or astronomy.

\section{Summary:}

The LCT-transformation is a immobility time synchronization between the two reference frames $\mathrm{A}$ and $\mathrm{B}$, where $\mathrm{A}$ is the absolute immobility reference frame, and $\mathrm{B}$ is the chaotic reference frame compared to $\mathrm{A}$. 
Immobility time synchronization equation has the form:

$$
\left\{\begin{array}{l}
t_{B}=\beta * t_{A}=\beta * T \\
\beta=1-\frac{v_{B}}{C_{\max }}
\end{array}\right.
$$

From the system of equation (5), we can deduce the distance value of the reference frame $\mathrm{B}$ when it moves in total time $\mathrm{T}$ :

$$
S_{B}=t_{B}^{\prime} * C_{\max }=T_{B} * v_{B}
$$

$S_{B}$ is the distance value that the frame of reference B travels, the motion graph of B is unknown, which means that orbit of B can be linear motion, or accelerated motion, or chaotic motion.

To apply the LCT-transformation into nature, we need to define an absolute reference frame, which means that we need to find the actual velocity of the earth (your or mine's velocity).

\section{Determine the real velocity of the earth}

There are two methods to find the real speed of the earth, the manuscript will present both ways and then combine them to produce specific numerical results that are not necessarily through experiment.(After calculating the specific values, the manuscript also proposed an optimal method to re-verify these values)

Note: Both methods are based on a proven experiment:

"The speed of light is constant for all inertial reference frames" This is also used as a axiom only for the manuscript to calculate the true velocity of the earth. 


\subsection{Method number 1}

Sir. Lorentz used this empirical evidence to construct Lorentz transformation for reference frame $\mathrm{B}$ corresponding to the velocity $v_{B}<v_{\text {light }}$.

So if we apply the real velocity of the earth $v_{B}\left(v_{B}=v_{E a r t h}\right)$ to the Lorentz transformation then it is perfectly valid, because if the velocity of the earth $v_{\text {Earth }} \geq v_{\text {light }}$ then the laws of physics will not be the same as it is now.

But because the origin of the time equation in the Lorentz transformation contains the distance variable of the reference systems, so we need to upgrade a bit to be able to use it in this manuscript.

Specifically, we will upgrade the time equation in the Lorentz transformation to the format:

$$
t_{B}=\sigma t_{A}
$$

Where: $\sigma$ is the new element of Lorentz, $\left(\sigma \neq \frac{1}{\sqrt{1-v^{2} / c^{2}}}\right) . \sigma$ is unknown, so it needs to be decoded.

Beginning decoding $\sigma$ :

Because the Lorentz transform is true for all inertial reference frames, it is also true for the absolute reference frame A.

$$
t_{B}=\sigma t_{A}=\sigma T_{B}
$$

On the other hand, we have:

$$
t_{B}=T_{B}-t_{B}^{\prime}
$$

Therefore:

$$
\begin{gathered}
\sigma T_{B}=T_{B}-t_{B}^{\prime} \\
\Leftrightarrow \sigma=1-\frac{t_{B}^{\prime}}{T_{B}} \Leftrightarrow \sigma=1-\sin ^{2}(x)
\end{gathered}
$$

We use trigonometry to make the intermediate object associated with the Lorentz transformation, which means that we need to find a value corresponding to the trigonometric function in the Lorentz transformation to assign it to the $\sigma$ value. 
And we find that the value $1-\frac{v_{B}^{2}}{v_{\text {light }}^{2}}$ is the value we need to look for in the Lorentz transformation, so:

$$
\sigma=1-\frac{v_{B}^{2}}{v_{\text {light }}^{2}}
$$

Finally, the immobility time of the two reference frames A and B in the Lorentz transformation is determined by the equations:

$$
\left\{\begin{array}{c}
t_{B}=\sigma * t_{A} \\
\sigma=1-\frac{v_{B}^{2}}{v_{\text {light }}^{2}}
\end{array}\right.
$$

And the immobility time of the two reference frames A and B in the LCTtransformation is determined by the equations:

$$
\left\{\begin{array}{l}
t_{B}=\beta * t_{A} \\
\beta=1-\frac{v_{B}}{C_{\max }}
\end{array}\right.
$$

Therefore, in the case of $v_{B}<v_{\text {light }}$, we have:

$$
\sigma=\beta \Leftrightarrow \frac{v_{B}^{2}}{v_{\text {light }}^{2}}=\frac{v_{B}}{C_{\max }} \Leftrightarrow v_{B}=\frac{v_{\text {light }}^{2}}{C_{\max }}
$$

Where:

- $v_{B}$ is the earth's real velocity, $v_{B}=v_{\text {Earth }}$

- $v_{\text {light }}$ is the speed of the light(vacuum) emitted from the earth's light source(light source of $B$ )

- $C_{\max }$ is the maximum velocity measured by the method mentioned in the immobility theory.

\subsection{Method number 2}

This method is a bit more complicated. First, the manuscript will prove a problem to get important consequences. 
The problem:

"Suppose there is an object $M$ inertial motion compared to the frame of reference $A$ ( $A$ is the absolute frame of reference), and the velocity of $M$ does not change compared to the inertial reference frame $B$ ( $B$ is inertial motion at any velocity compared to $A$ ). What to prove: The velocity of $M$ is the maximum velocity $v_{M}=C_{\max }$ "

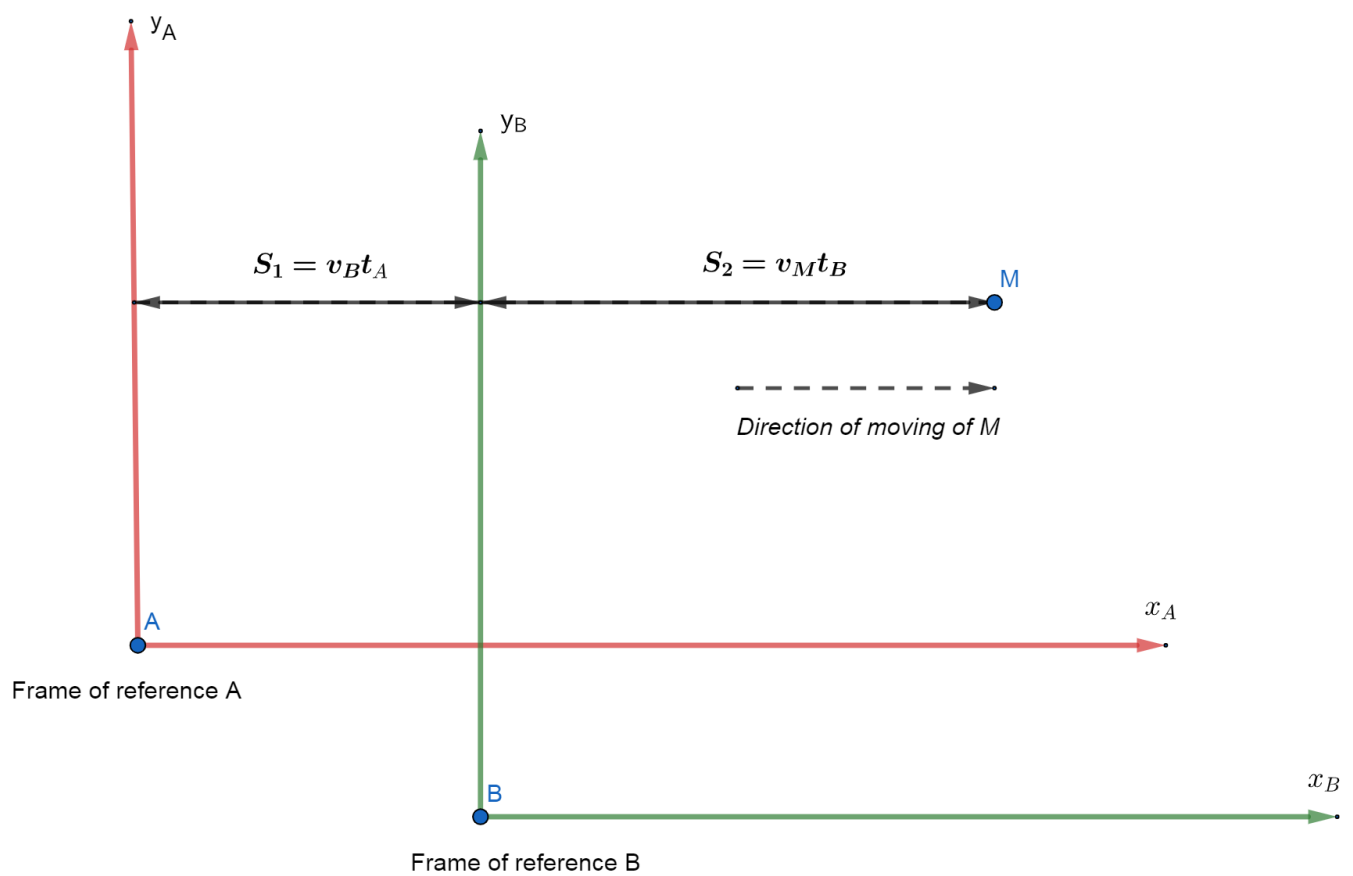

(Figure 3: Prove that the velocity of $M$ reaches maximum)

Proving:

We have:

$$
S_{1}+S_{2}=v_{B} * t_{A}+v_{M} * t_{B}=C_{\max } * t_{B}^{\prime}+v_{M} * t_{B}
$$


On the other hand, we have:

$$
S_{1}+S_{2}=v_{M} * t_{A}=v_{M} * T
$$

Therefore, we get the equation:

$$
v_{M} * T=C_{\max } * t_{B}^{\prime}+v_{M} * t_{B}
$$

Analyze the right side of the equation (7):

- If $v_{M} \neq C_{\max }$ then:

$$
v_{M} * t_{B}^{\prime}+v_{M} * t_{B}<C_{\max } * t_{B}^{\prime}+v_{M} * t_{B} \Leftrightarrow v_{M} * T<C_{\max } * t_{B}^{\prime}+v_{M} * t_{B}
$$

- Hence the equation (7) is only true if and only if $v_{M}=C_{\max }$

Proving end.

Based on the results of the proven problem, now, we will use a series of reasoning methods to get important consequences.

$\underline{\text { Begin reasoning: }}$

- Experiments have shown that the speed of light is always constant for all inertial reference frames, so if we replace object $M$ with light in the proven problem then we get $v_{\text {light }}=C_{\max }(?)$

- This is very strange, because if $v_{\text {light }}=C_{\max }$ then when we replace these values into equation (6), we get the velocity $v_{B}$ of the earth with $C_{\max }$ :

$$
v_{B}=\frac{v^{2} \text { light }}{C_{\max }}=\frac{C^{2}{ }_{\max }}{C_{\max }}=C_{\max }=v_{\text {Earth }}
$$


- This is not possible, because as we all know that the velocity $v_{B}$ of the earth is not the maximum in the universe.

- Therefore, there is a mystery going on here.

Continue reasoning:

- We will approach the problem in a different direction: The speed of light is always emitted from the light source, while the light source is considered to be a moving reference frame (not an absolute immobility reference frame).

- Therefore, to satisfy the logic of the problem and not allowed to appear contradictory then:

$$
v_{\text {light_source }}+v_{\text {light }}=C_{\max }
$$

- This means that velocity of light $v_{\text {light }}$ always depends on the velocity of light source $v_{\text {light_source }}$, and any other inertial reference frame when measuring that speed of light then it is necessary to add the speed of the light source $v_{\text {light_source }}$ to create a maximum value $C_{\max }$ as mentioned in equation (8).

Right now, we will prove equation (8) is correct and logical with the axiom of the manuscript. This means that we will prove that if velocity of light depends on the light source then its velocity remains still unchanged from all inertial reference frames.

Proving:

Suppose Alice is on an airplane and emit a light from her light source, and Bob on the ground will measure the speed of light with his watch $\left(v_{\text {light_of_Bob }}\right)$. 
Of course, Alice also measures the speed of light with her watch $\left(v_{\text {light_of_Alice }}\right)$.

Then the speed of light obtained from Alice and Bob's measurements is calculated as follows:

- Speed difference between Alice and Bob:

$$
\Delta v=v_{\text {Alice }}-v_{\text {Bob }}
$$

- The value of $\Delta v$ is also the value of light speed difference of Alice and Bob:

$$
\Delta v=v_{\text {light_of_Bob }}-v_{\text {light_of_Alice }}
$$

- The value of speed of light that Alice measured from her frame of reference:

$$
\begin{gathered}
v_{\text {light_of_Alice }}=C_{\max }-v_{\text {Alice }}=C_{\max }-v_{B o b}-\Delta v \\
\Rightarrow C_{\max }=v_{\text {light_of_Alice }}+v_{B o b}+\Delta v
\end{gathered}
$$

- On the other hand, the value of speed of light that Bob measured from his frame of reference:

$$
\begin{aligned}
& v_{\text {light_of_Bob }}=C_{\max }-v_{B o b} \\
\Rightarrow & C_{\max }=v_{\text {light_of_Bob }}+v_{B o b}
\end{aligned}
$$

- Argument: If the $C_{\max }$ values of equations $(9)$ and $(10)$ are equal then we have proven success. Conversely, if the $C_{\max }$ values of the two equations are different then the speed of light will be different for each inertial reference frame.

- Therefore, assuming $C_{\max }$ values are equal in both equations (9) and (10), we have:

$$
v_{\text {light_of_Alice }}+v_{B o b}+\Delta v=v_{\text {light_of_Bob }}+v_{B o b}
$$




$$
\begin{gathered}
\Leftrightarrow v_{\text {light_of_Alice }}+\Delta v=v_{\text {light_of_Bob }_{-}} \\
\Leftrightarrow 0=0
\end{gathered}
$$

Proving end.

Conclusion: The real speed of light depends on the speed of the light source. This does not mean that the speed of light is maximum, but that it is always at its maximum when combined with the speed of the reference frame of its source.

$$
v_{\text {light_source }}+v_{\text {light }}=C_{\max }
$$

\subsection{The combination of two methods}

Combining equation (6) in method number 1 and equation (8) in method number 2, we get:

$$
v_{\text {Earth }}=\frac{v_{\text {light_earth }}^{2}}{C_{\max }}=C_{\max }-v_{\text {light_earth }}
$$

Solving Equation (11), we get the following important results:

$$
\frac{C_{\text {max }}}{v_{\text {light_earth }}}=\frac{1+\sqrt{5}}{2}=\varphi=\text { Golden_ratio }
$$

And:

$$
\frac{v_{\text {light_earth }}}{v_{\text {Earth }}}=\varphi=\text { Golden_ratio }
$$

Substituting specific values into the equations $(12)$ and $(13)$, we get:

$$
v_{\text {Earth }}=\frac{v_{\text {light }}}{\varphi}=\frac{2.99792458 * 10^{8}}{1.61803398}=1.852819296 * 10^{8} \mathrm{~m} / \mathrm{s}
$$

And:

$$
C_{\max }=\varphi * v_{\text {light }}=4.8507438399 * 10^{8} \mathrm{~m} / \mathrm{s}
$$




\section{The consequences:}

- The speed of light depends on the light source and also the maximum speed in the universe, the dependence of the speed of light is described by the golden ratio as follows:

$$
\varphi=\frac{C_{\max }}{v_{\text {light }}}=\frac{v_{\text {light }}}{v_{\text {light_source }}}
$$

- The $C_{\max }$ value we measure on earth is also not a constant for the whole universe, because as the velocity of the light source $v_{\text {light_source }}$ increases, both $C_{\max }$ and $v_{\text {light }}$ also increase to follow the golden ratio.

\subsection{Proposing method of verification}

- We have a method of measuring $C_{\max }$ in the immobility theory. If the result of $C_{\max }$ after measurement are consistent with the ratios mentioned in this manuscript then the manuscript results are correct.

- In addition, we have another evidence that we cannot accelerate an object to achieve velocity by $v_{\text {light }}$. The reason for this phenomenon is that our earth's reference frame is moving at a speed $v_{\text {Earth }}=C_{\max }-v_{\text {light_earth. Therefore, all }}$ objects in motion on the earth must add a velocity to its frame of reference, which is the velocity $v_{\text {Earth }}=1.852819296 * 10^{8} \mathrm{~m} / \mathrm{s}$.

\section{References}

[1] Immobility theory - doi.org

[2] Speed of light - Wikipedia

[3] Lorentz transformation - Wikipedia 